\title{
Die Amputation nach Pirogow als Alternative zur Unterschenkelamputation?
}

\author{
Pirogow's Amputation as an Alternative to Lower Leg Amputation?
}

\author{
Autoren \\ M. Bueschges ${ }^{1}$, C. Ottomann ${ }^{1}$, K. Mauss ${ }^{1}$, T. Muehlberger ${ }^{2}$, J. C. Bruck ${ }^{3}$ \\ Institute \\ ${ }^{1}$ Sektion für Plastische Chirurgie und Handchirurgie, Intensiveinheit für Schwerbrandverletzte, Universitätsklinikum \\ Schleswig Holstein Campus Lübeck, Lübeck \\ ${ }^{2}$ MCZ, Dep. of Plastic Surgery, Berlin \\ ${ }^{3}$ Plastische Chirurgie, Martin-Luther-Krankenhaus, Berlin
}

\author{
Schlüsselwörter \\ - Amputation \\ - Pirogow \\ - Unterschenkelamputation \\ - Ankle Score
}

Key words

- amputation

- Pirogow

- lower leg amputation

- ankle score

eingereicht 13.9.2012

akzeptiert $\quad 11.12 .2012$

\section{Bibliografie \\ DOI http://dx.doi.org/ \\ 10.1055/s-0032-1333270 \\ Online-Publikation: 6.3.2013 \\ Handchir Mikrochir Plast Chir \\ 2013; 45: 67-72 \\ (c) Georg Thieme Verlag KG \\ Stuttgart · New York \\ ISSN 0722-1819}

\section{Korrespondenzadresse \\ Dr. Michael Bueschges}

Sektion für Plastische Chirurgie und Handchirurgie

Intensiveinheit für

Schwerbrandverletzte

Universitätsklinikum Schleswig

Holstein Campus Lübeck

Ratzeburger Allee 160

23560 Lübeck

michaelbueschges@gmx.de

\section{Zusammenfassung \\ $\nabla$}

Die Amputation nach Pirogow auf Höhe des Sprunggelenkes stellt bei entsprechender Vorraussetzung eine wertvolle Alternative zur Unterschenkelamputation dar. Hierbei handelt es sich um eine Amputation auf Höhe des oberen Sprunggelenkes, jedoch unter Erhalt des Tuber calcanei und eines Teils der Fersenhaut als sensible, stabile Belastungszone für den Stumpf. Bei der Pirogow Operation wird das mit der Fersenhaut erhaltene Tuber calcanei - nach Extirpation des restlichen Calcaneus, des Talus und der supramalleoläre quer osteotomierten Knöchelgabel - um ca. $70^{\circ}$ nach oben geklappt und der Tibiaosteotomie unterstellt. Der operative Zugang für die Amputation erfolgt von vorn (ventral), mit Bildung eines weiter nach distal reichenden Sohlenlappens, der dann den ventralen Defekt decken kann und auch dort eine Endbelastungsfähigkeit herstellt $[6,10]$. Obwohl sie für den Patienten den Vorteil bietet, ohne Prothese mobil zu sein, wird sie selten durchgeführt (0,1\% der Amputationen der unteren Extremität). Bei 20 Patienten mit durchgeführter Pirogowschen Amputation wurden 12 Monate nach der Operation die Ergebnisse anhand des Ankle Score Patientenfragebogens von Taniguchi et al. bewertet. Durch ein Punktesystem wurden die Kriterien Schmerz, funktionelle und radiologische Beurteilung, Beinlängendifferenz und Mobilität ohne Prothese objektiviert. Ein identischer Fragebogen wurde 20 Patienten, bei denen eine Unterschenkelamputation durchgeführt wurde, in gleichem Zeitabstand nach 12 Monaten vorgelegt. Ein exzellentes bzw. gutes Ergebnis gaben nach Pirogowscher Amputation 65\% an, in der Vergleichsgruppe nach Unterschenkelamputation gaben $60 \%$ ein exzellentes bzw. gutes Ergebnis an. Bei der Pirogow Gruppe trat bei 30\% der Patienten eine Komplikation auf, die einen Revisionseingriff notwendig machten, gegenüber

\section{Abstract \\ $\nabla$}

Pirogow's amputation at the ankle presents a valuable alternative to lower leg amputation for patients with the corresponding indications. Although this method offers numerous advantages for the patient, such as the ability to stay mobile without the use of a prosthesis, it is rarely performed $(0.1 \%$ of all lower limb amputations). The results of the operations on 20 patients were objectified 12 months after the operation using a patient questionnaire (Ankle Score), and these results were then compared to those of 20 patients who underwent lower leg amputation. Using a point system the criteria pain, functional and radiological assessment, difference in leg length, and mobility without prosthesis were recorded and evaluated. $65 \%$ of those questioned who were amputated following the Pirogow method indicated an excellent or very good result, in the control group $60 \%$ of those having undergone a lower leg amputation responded similarly, indicating an excellent or very good result.

In $30 \%$ in the Pirogow group in contrast to $20 \%$ after lower leg amputation postoperative complications lead to a revision-operation. In patients suffering from diabetes or restricted perfusion of the lower extremity an amputation at the level of the ankle has to be considered critically keeping the necessity of a revision-operation in mind. However, if it can be carried out successfully, the benefits of Pirogow-amputation are found in the significantly reduced difference in leg length and the increase in mobility without prosthesis. 
20\% der Patienten nach Unterschenkel Amputation. Bei Diabetikern oder Patienten mit mangelhafter Durchblutungssituation der unteren Extremität ist die Amputation auf Höhe des Sprunggelenkes kritisch zu betrachten und sollte nur nach Abwägung des Risikos eines Revisionseingriffes erfolgen. Kann jedoch eine erfolgreich durchgeführte Amputation auf Höhe des Sprunggelenkes erreicht werden, stellt dies für den Patienten aufgrund der Mobilität ohne Prothese, der einfacheren prothetischen Versorgung und der geringeren zu leistenden Geharbeit, einen wesentlichen Vorteil dar.

\section{Einleitung}

In seinem Originalartikel aus dem Jahre 1854 berichtete Nikolai Iwanowitsch Pirogow über mehrere 100 durchgeführte Amputationen der unteren Extremität, welche er während des Krimkrieges durchgeführt hatte. Er versuchte die aufgrund der häufig auftretenden Fußverletzungen - militärhistorisch durch die zunehmende Verwendung von Minen - notwendigen Unterschenkelamputationen zu umgehen [7]. Pirogow entwickelte eine Amputationsmethode im Sprunggelenk, welche für den Patienten eine Vielzahl von Vorteilen gegenüber der bisher durchgeführten Unterschenkelamputation besaß [12]. Ein wesentlicher Vorteil bestand in der deutlich verringerten Beinlängendifferenz, der eine Mobilisierung ohne Prothese erlaubte und damit - insbesondere in der damaligen Zeit der mangelhaften prothetischen Versorgung - einen geringeren Behinderungsgrad für den Patienten bedeutete $\triangle$ Abb. 1. Ob die Amputation nach Pirogow heute noch vorteilhaft ist, wurde bei 20 Patienten durch einen Patientenfragebogen eruiert, dabei wurden die Ergebnisse mit einer Vergleichsgruppe bestehend aus 20 Patienten, bei denen eine Unterschenkelamputation durchgeführt wurde, verglichen. Dieser Untersuchung sollte eine vergleichende retrospektive Fallkontrollstudie folgen, die die Ergebnisse der Pirogow Technik den Ergebnissen der Unterschenkel Amputation gegenüberstellt.

\section{Die Modifikation der Rückfuß Amputation nach Pirogow}

Bei der Pirogow Originalmethode tritt oftmals das Problem auf, dass das Adaptieren der Osteotomieflächen schwierig ist. Die Originalmethode hat daher zahlreiche Modifikationen erfahren. Günther et al. haben vorgeschlagen, den Knochen von schräg hinten nach vorn unten zu durchtrennen, um die Adaptionsoberfläche zu erhöhen [10]. Le Fort und andere modifizierten diese Methode erneut und durchtrennten den Kalkaneus horizontal $[2,10]$. Im Gegensatz dazu ist unter Anderem von Tauber und Eisenberg beschrieben, den Kalkaneus sagittal zu mobilisieren [10]. Weitere Modifikationen sind außerdem beispielsweise von Spitzy, Küster, Richard, Boydt, Jacob oder Borchard beschrieben $[2,5,10]$.

In dieser Arbeit wurde bei den untersuchten Patienten eine im Folgenden beschriebene Methode verwendet: nachdem die Weichteile bis auf den Knochen durchtrennt sind, wird der Vorfuß mitsamt dem Talus scharf vom Kalkaneus getrennt. Sodann wird die vordere Gelenkfläche zum Cuboid abgesägt und die mittlere und hintere Gelenkfläche des Kalkeneus zum Talus in einer Achse von etwa $30^{\circ}$ zur Längsachse des Kalkaneus abgesetzt $($ Abb. 2, 3). Auch die Gelenkfläche der Tibea wird mit der Säge begradigt, und beidseits werden im Winkel von etwa $45^{\circ}$ der mediale und laterale Malleolus abgesetzt. Um den Rotationsbogen des Kalkaneus auf eine plane Ebene der tibiotalaren Gelenkfläche einschwenken zu können, wurde die Achillessehne oberhalb des Kalkaneus scharf durchtrennt. Lässt sich der Kalkaneus gut auf die distale Tibea einstellen, wird dieser mit 2 gekreuzten Bohrdrähten in Position fixiert. Über die Bohrdrähte wurden 2,4 mm dicke Hohlschrauben eingeführt um so das Kalkaneusfragment an die Tibea zu fixieren. Es wurde darauf geachtet, dass der Kreuzungspunkt der Schrauben innerhalb des

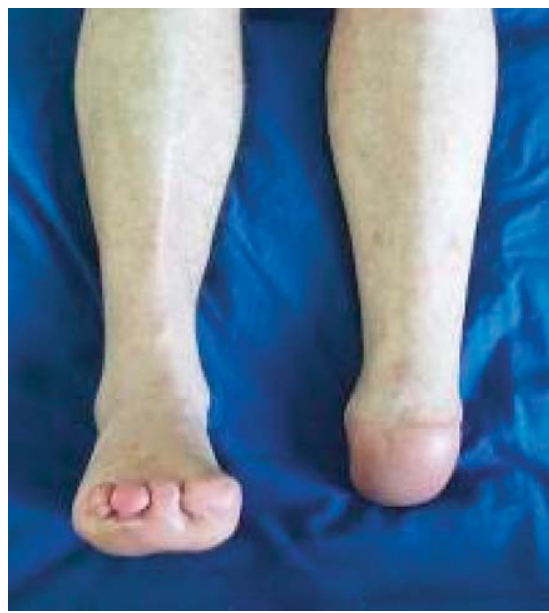

Abb. 1 Exemplarische Darstellung der Beinlängendifferenz zwischen amputierten und gesunden Bein (Gruppe $1=\varnothing \mathrm{ca}$. $2,8 \mathrm{~cm}$. Gruppe $2=\varnothing$ $1,9 \mathrm{~cm})$.

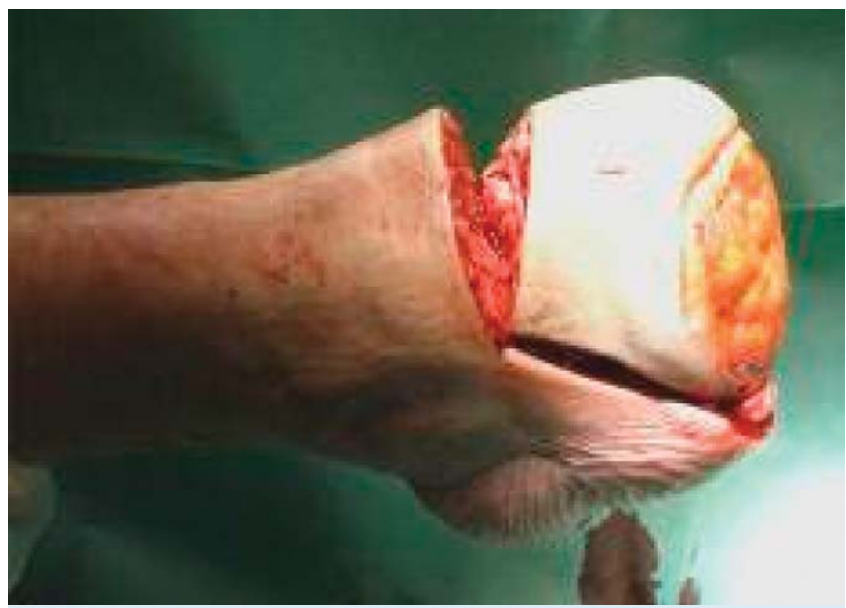

Abb. 2 Intraoperatives Bild nach Inzision der Schnittränder und der Gelenkfläche und des oberen Sprunggelenkes.

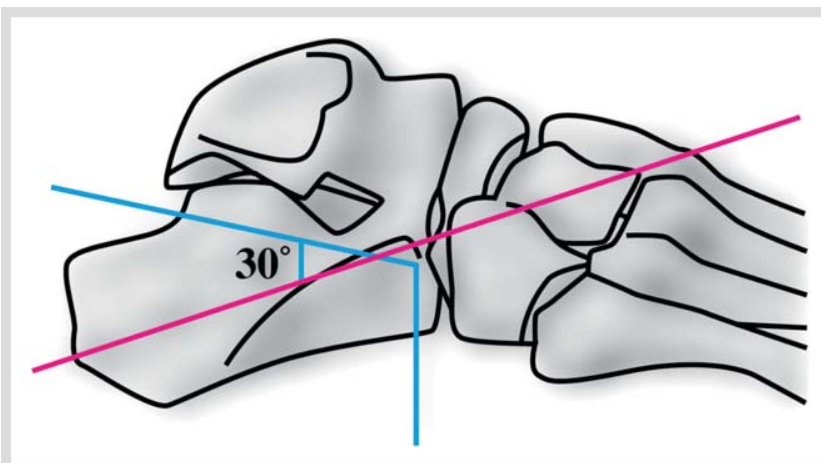

Abb. 3 Die mittlere und hintere Gelenkfläche wird in einer Achse von etwa $30^{\circ}$ zur Längsachse des Kalkaneus abgesetzt. 
Tab. 1 Modifizierter Ankle and Hindfoot score (AOFAS) mit Berücksichtigung fehlender Funktion im Sprunggelenk und mehr Gewicht auf funktionelle und radiologische Kriterien $\mathrm{n}$. Taniguchi.

\begin{tabular}{|c|c|c|c|c|}
\hline \multirow{2}{*}{$\begin{array}{l}\text { Kriterium } \\
\text { Schmerz }\end{array}$} & \multicolumn{4}{|c|}{ Ausprägung/Punktwert } \\
\hline & Schmerzlos (40) & Leicht (30) & Moderat (20) & Stark (10) \\
\hline \multicolumn{5}{|l|}{ Funktionelle Kriterien } \\
\hline Gehstrecke & uneingeschränkt (20) & 2 km (15) & $500 m-2 \mathrm{~km}(10)$ & häuslich (5) \\
\hline Hinken & & kein Hinken (4) & moderat (2) & gehunfähig (0) \\
\hline Treppaufsteigen & & uneingeschränkt (4) & am Geländer (2) & N.m. (0) \\
\hline Treppabsteigen & & uneingeschränkt (4) & am Geländer (2) & N.m. (0) \\
\hline Einbeinstand & & uneingeschränkt (4) & Unterstützung (2) & N.m. (0) \\
\hline Schneidersitz & & uneingeschränkt (4) & mit Unterstützung (2) & N.m. (0) \\
\hline \multicolumn{5}{|l|}{ Radiologische Kriterien } \\
\hline Knochenatrophie & & keine (5) & Trabekelatrophie (3) & Nekrose (0) \\
\hline plantare Weichteile & & $>2 \mathrm{~cm}(5)$ & $1-2 \mathrm{~cm}(3)$ & $<1 \mathrm{~cm}(0)$ \\
\hline Beinlängendifferenz & & keine (5) & $<2 \mathrm{~cm}(3)$ & $>2 \mathrm{~cm}(0)$ \\
\hline Mob. ohne Prothese & & uneingeschränkt (5) & $>10 \mathrm{~m}(3)$ & $<10 \mathrm{~m}(0)$ \\
\hline Gesamtpunkte (100) & exzellent (>80) & gut (60-79) & befriedigend (40-59) & schlecht $(<40)$ \\
\hline
\end{tabular}

Kalkaneus in Lage kam. Die Gelenkflächen wurden möglichst so gewählt, dass der Kalkaneus in seiner Achse etwa $15^{\circ}$ VarusStellung zur Tibea-Achse einnimmt.

\section{Material und Methoden}

In den Jahren 2000-2008 wurden in der Abteilung für Plastische Chirurgie im Martin-Luther-Krankenhaus Berlin 37 Patienten durch verschieden modifizierte Rückfuß Amputationen nach Pirogow versorgt [9]. 20 der insgesamt 37 Patienten wurden mittels der oben beschriebenen Operation versorgt und konnten 12 Monate nach der Amputation in die Studie eingeschlossen und mit einem Fragebogen bewertet werden. Der Fragebogen ist an den Ankle and Hindfoot Score der Amerikanischen Gesellschaft für Fuß und Sprunggelenke (AOFAS) angelehnt und legt Gewicht auf funktionelle und radiologische Kriterien $\bullet$ Tab. 1. In einem Punktesystem (0-100 Punkte) werden verschiedene Kriterien zur Bewertung des Ergebnisses und der resultierenden Funktion benutzt: Schmerz, funktionelle und radiologische Kriterien, Beinlängendifferenz sowie die Mobilität ohne Prothese sind durch einen bestimmten Punktwert gekennzeichnet. Die funktionellen Kriterien bei Tragen der Prothese gliedern sich in Gehstrecke, Hinken, der Fähigkeit des Treppauf- und Treppabsteigens, des Einbeinstandes und der Fähigkeit des Schneidersitzes. Die radiologischen Punkte werden durch Zeichen der Knochenatrophie und Bewertung des plantaren Weichteilmantels im Nativ Röntgen des Amputationsstumpfes vergeben ( $\bullet$ Abb. 4). Die Punkteverteilung der Beinlängendifferenz zwischen gesundem Bein und Amputationsstumpf richtet sich nach der Länge in cm, die Mobilität ohne Prothese nach der Gehstrecke. Bei einem maximal erreichbarem Wert von 100 Punkten stellt ein Wert größer als 80 Punkte ein exzellentes Ergebnis dar, ein Wert zwischen 60-79 Punkten ein gutes, zwischen 40-59 Punkten ein befriedigendes und ein Resultat kleiner 40 Punkten ein schlechtes Ergebnis dar $\bullet$ Tab. 1. Der Ankle Score Fragebogen wurde den Patienten 12 Monate (plus/minus 2 Wochen) nach durchgeführter Rückfuß Amputation nach Pirogow vorgelegt. 20 Patienten, bei denen eine Unterschenkelamputation durchgeführt wurde, wurde ein identischer Fragebogen nach gleichem postoperativem Zeitabstand als Vergleichsgruppe vorgelegt (12 Monate Follow-Up).

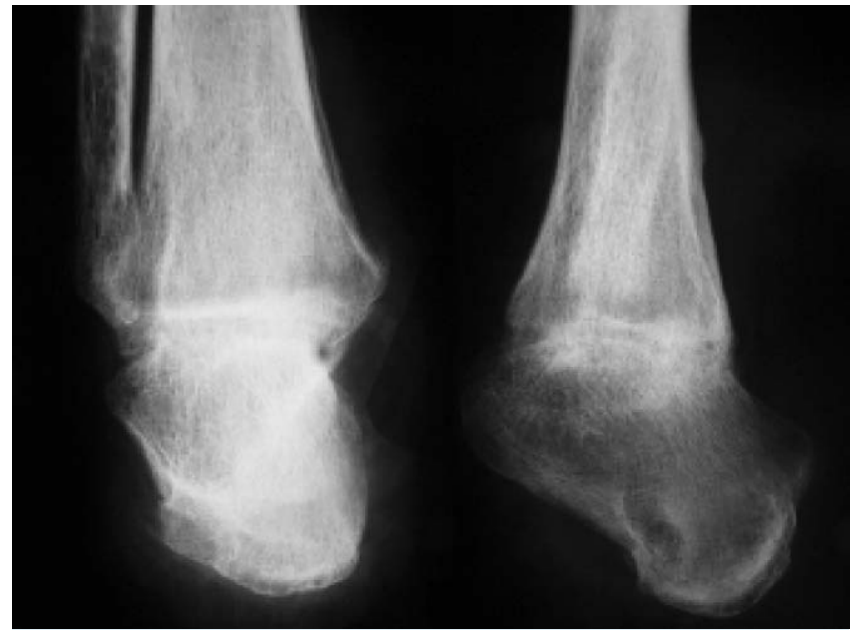

Abb. 4 Radiologische Kontrollaufnahme 6 Monate postoperativ (Gruppe 1).

\section{Ergebnisse \\ $\nabla$}

\section{Gruppe 1 = Pirogow Amputation}

Das Durchschnittsalter der Patientengruppe mit durchgeführter Pirogowscher Amputation betrug 65,5 Jahre. 15 Patienten waren männlich (75\%), 5 weiblich (25\%). Bei insgesamt 6 Patienten (30\%) resultierte eine Wundheilungsstörung. Die Grunderkrankungen und Indikationen, die zur Amputation nach Pirogow führten, sind in $\bullet$ Tab. 2 aufgeführt. Alle 6 Patienten, die von einer Komplikation betroffen waren, litten unter Diabetes mellitus. Art der Komplikation und die daraus resultierenden Revisionseingriffe finden sich ebenfalls in $\bullet$ Tab. 2. Eine verzögerte Wundheilung wurde nur dann als Komplikation gewertet, wenn diese zu einem notwendigen Revisionseingriff führte.

Die Auswertung der Gruppe 1 nach dem Ankle Score (AS) resultierte in den in Tab. 3, 4 dargestellten Punktwerten. In 20\% resultierte ein exzellentes, in $45 \%$ ein gutes Ergebnis 12 Monate nach der Operation. Spezifiziert man das Gesamtergebnis der einzelnen Unterfelder des AS, so zeigt sich bezüglich des Schmerzes folgende Verteilung: 6 Patienten (30\%) gaben im Follow-Up 12 Monate nach der Operation im Pirogow Stumpf keine Schmerzen an (schmerzlos), 6 Patienten (30\%) leichte Schmerzen, 4 Patienten (20\%) moderate und 4 Patienten (20\%) starke 
Tab. 2 Grunderkrankungen, Indikationen, Komplikationen und Anzahl der Grunderkrankungen Gruppe 1 (Pirogow).

\begin{tabular}{|lllll} 
Patient & Grunderkrankung & Indikation & Komplikation & Anzahl der Revisionseingriffe \\
\hline 1 & Nikotinabusus, PAVK & Ulcus cruris & keine & 0 \\
\hline 2 & keine & Überrolltrauma & keine & 0 \\
\hline 3 & Diabetes mellitus & Gangrän & keine & 0 \\
\hline 4 & Diabetes mellitus & diabetisches Ulcus & Wundheilungsstörung & 1 (Spalthauttransplantation) \\
\hline 5 & postthrombotisches Syndrom & chronische Wunde & keine & 0 \\
\hline 6 & Melanom & Melanom & keine & 0 \\
\hline 7 & Diabetes mellitus & Ulcus cruris & Wundheilungsstörung & 1 (Nachamputation Höhe US) \\
\hline 8 & Morbus Raynaud & Nekrose & keine & 0 \\
\hline 9 & Nikotin und Alkoholabusus, PAVK & Ulcus cruris & verzögerte Wundheilung & 0 \\
\hline 10 & Alkoholabusus & Erfrierung & keine & 0 \\
11 & Diabetes mellitus & Nekrose & Nekrose & 2 (Spalthauttransplantation) \\
\hline 12 & keine & Quetschtrauma & keine & 0 \\
\hline 13 & PAVK & Gangrän & verzögerte Wundheilung & 0 \\
\hline 14 & Diabetes mellitus & diabetisches Ulcus & Nekrose & 1 (Nachamputation Höhe US) \\
\hline 15 & PAVK & Ulcus cruris & keine & 0 \\
\hline 16 & Diabetes mellitus & Nekrose & Wundheilungsstörung & 1 (Spalthauttransplantation) \\
\hline 17 & Diabetes mellitus & diabetisches Ulcus & verzögerte Wundheilung & 0 \\
\hline 18 & Diabetes mellitus & Gangrän & Wundnekrose & 3 (Nachdebridement, Nachamputation Höhe US) \\
\hline 19 & Kontaktverbrennung & Verbrennung Grad III & keine & 0 \\
\hline 20 & PAVK & Ulcus cruris & keine & 0 \\
\hline & & & & \\
\hline
\end{tabular}

Schmerzen. Ein gutes funktionelles Ergebnis resultierte 12 Monate postoperativ bei 12 Patienten (60\%). In 30\% zeigten sich Zeichen der Knochenatrophie in der radiologischen Diagnostik der Adaptionsstelle zwischen Tibia und Kalkaneus. Bezüglich der Beinlängendifferenz zeigte sich in unserer Untersuchung bei einem Patienten keine Differenz (5\%), bei 4 Patienten eine Differenz $<2 \mathrm{~cm}$ (20\%) und bei 15 Patienten eine Beinlängendifferenz von $>2 \mathrm{~cm}$ (75\%). Durchschnittlich resultierte eine Differenz von $2,9 \mathrm{~cm}$. Die Mobilität ohne Prothese war bei 2 Patienten uneingeschränkt (10\%), bei 12 Patienten $(60 \%)>10 \mathrm{~m}$, bei 6 Patienten betrug die Gehstrecke ohne Prothese $<10 \mathrm{~m}$ (30\%).

\section{Gruppe 2 = Unterschenkelamputation}

In der Vergleichsgruppe der Patienten mit durchgeführter Unterschenkelamputation betrug das Durchschnittsalter 61,45 Jahre. 12 Patienten (60\%) waren männlich, 8 Patienten (40\%) waren weiblich. Die Grunderkrankungen und Indikationen, die zur Unterschenkel Amputation führten, sind in $\bullet$ Tab. 7 aufgeführt. Bei insgesamt 4 Patienten (20\%) resultierte eine Wundheilungsstörung. Alle 4 Patienten, die von einer Wundheilungsstörung betroffen waren, litten unter Diabetes mellitus. Die Art der Komplikation und die daraus resultierenden Revisionseingriffe finden sich ebenfalls in $\bullet$ Tab. 7. Eine verzögerte Wundheilung wurde nur dann als Komplikation gewertet, wenn diese zu einem notwendigen Revisionseingriff führte.

Die Auswertung nach dem Ankle Score (AS) bei der Patientengruppe, die mittels Unterschenkel Amputation behandelt wurde, wird in den in $\bullet$ Tab. 5, 6 dargestellten Punktwerten dargestellt. In $10 \%$ resultierte ein exzellentes, in 50\% ein gutes Ergebnis. Spezifiziert man das Gesamtergebnis der einzelnen Unterfelder des AS, so zeigt sich bezüglich des Schmerzes, der nach der Unterschenkel Amputation auftritt, folgende Verteilung: 7 Patienten (35\%) gaben im Follow-Up 12 Monate nach der Unterschenkel Amputation an keine Schmerzen zu haben (schmerzlos), 5 Patienten (25\%) leichte Schmerzen, 5 Patienten (30\%) moderate und 2 Patienten (10\%) starke Schmerzen. Ein gutes funktionelles Ergebnis ergab sich 12 Monate postoperativ bei 12 Patienten (60\%). Bezüglich der Beinlängendifferenz ergab sich
Tab. 3 Einzelergebnisse Gruppe 1 (Pirogow).

\begin{tabular}{|rllrlll|} 
Patient & Schmerz & Funktion & Radiologie & LD & $\begin{array}{l}\text { Mobilität } \\
\text { o.P. }\end{array}$ & Gesamt \\
\hline 1 & 40 & 25 & 6 & 3 & 0 & 74 gut \\
\hline 2 & 30 & 36 & 10 & 3 & 3 & 82 exz. \\
\hline 3 & 40 & 27 & 8 & 3 & 3 & 81 exz. \\
\hline 4 & 30 & 23 & 3 & 3 & 3 & 62 gut \\
\hline 5 & 30 & 32 & 8 & 3 & 3 & 76 gut \\
\hline 6 & 20 & 15 & 3 & 0 & 0 & 38 schl. \\
\hline 7 & 40 & 25 & 6 & 3 & 3 & 77 gut \\
\hline 8 & 40 & 29 & 8 & 3 & 3 & 83 exz. \\
\hline 9 & 20 & 14 & 6 & 3 & 0 & 33 schl. \\
\hline 10 & 10 & 18 & 6 & 0 & 0 & 34 schl. \\
\hline 11 & 40 & 27 & 6 & 3 & 3 & 79 gut \\
\hline 12 & 30 & 14 & 10 & 0 & 5 & 49 bef. \\
\hline 13 & 40 & 28 & 3 & 3 & 0 & 74 gut \\
\hline 14 & 10 & 9 & 3 & 0 & 0 & 22 schl. \\
\hline 15 & 20 & 16 & 0 & 3 & 0 & 39 schl. \\
\hline 16 & 30 & 35 & 5 & 3 & 3 & 76 gut \\
\hline 17 & 40 & 16 & 3 & 0 & 3 & 62 gut \\
\hline 18 & 40 & 40 & 10 & 3 & 3 & 96 exz. \\
\hline 19 & 30 & 35 & 5 & 3 & 3 & 76 gut \\
\hline 20 & 20 & 14 & 0 & 3 & 0 & 37 schl. \\
\hline
\end{tabular}

Tab. 4 Ergebnisse Gruppe 1 (Pirogow).

\begin{tabular}{|lllc|}
\hline Punktwert & Ergebnis & Anzahl & Prozent \\
$>80$ & exzellent & 4 & $20 \%$ \\
$60-79$ & gut & 9 & $45 \%$ \\
\hline $40-59$ & befriedigend & 1 & $5 \%$ \\
\hline$<40$ & schlecht & 6 & $30 \%$ \\
\hline
\end{tabular}

als Nachteil der Unterschenkelamputation grundsätzlich eine Differenz $>2 \mathrm{~cm}$ entsprechend einem variablem Punktwert von null Punkten. Ebenso ist eine Mobilität ohne Prothese bei Unterschenkel amputierten Patienten nur sehr begrenzt möglich, auch das wurde so als nicht variabler Punktwert mit null Punkten bewertet. 


\section{Diskussion}

Obwohl die Amputation nach Pirogow zahlreiche Vorteile für den Patienten bietet, allen voran die Möglichkeit, kurze Strecken auch ohne Prothese und geringer Geharbeit mobil zu sein, wird sie sehr selten durchgeführt (0,1\% der Amputationen der unteren Extremität) [13]. Die Indikation der Amputation nach Pirogow kann gestellt werden in Fällen, wo eine suffiziente Durch-

Tab. 5 Einzelergebnisse Gruppe 2 (Unterschenkelamputation).

\begin{tabular}{|rrrrrrl}
\hline Patient & Schmerz & Funktion & Radiologie & LD & $\begin{array}{l}\text { Mobilität } \\
\text { o. P. }\end{array}$ & Gesamt \\
\hline 1 & 20 & 36 & 8 & 0 & 0 & 64 gut \\
\hline 2 & 40 & 19 & 10 & 0 & 0 & 69 gut \\
\hline 3 & 30 & 20 & 8 & 0 & 0 & 58 bef. \\
\hline 4 & 30 & 28 & 8 & 0 & 0 & 64 gut \\
\hline 5 & 10 & 14 & 0 & 0 & 0 & 24 schl. \\
\hline 6 & 20 & 32 & 3 & 0 & 0 & 56 bef. \\
\hline 7 & 40 & 40 & 10 & 0 & 0 & 90 exz. \\
\hline 8 & 40 & 16 & 5 & 0 & 0 & 61 gut \\
\hline 9 & 10 & 23 & 8 & 0 & 0 & 41 bef. \\
\hline 10 & 40 & 27 & 10 & 0 & 0 & 77 gut \\
\hline 11 & 30 & 32 & 8 & 0 & 0 & 70 gut \\
\hline 12 & 40 & 20 & 3 & 0 & 0 & 63 gut \\
\hline 13 & 20 & 24 & 3 & 0 & 0 & 47 bef. \\
\hline 14 & 20 & 14 & 5 & 0 & 0 & 39 schl. \\
\hline 15 & 30 & 36 & 10 & 0 & 0 & 76 gut \\
\hline 16 & 40 & 36 & 8 & 0 & 0 & 84 exz. \\
\hline 17 & 10 & 19 & 3 & 0 & 0 & 32 schl. \\
\hline 18 & 10 & 5 & 5 & 0 & 0 & 20 schl. \\
\hline 19 & 30 & 28 & 3 & 0 & 0 & 61 gut \\
\hline 20 & 20 & 32 & 10 & 0 & 0 & 62 gut \\
\hline
\end{tabular}

Tab. 6 Ergebnisse Gruppe 2 (Unterschenkelamputation).

\begin{tabular}{|llcl|}
\hline Punktwert & Ergebnis & Anzahl & Prozent \\
\hline$>80$ & exzellent & 2 & $10 \%$ \\
\hline $60-79$ & gut & 10 & $50 \%$ \\
\hline $40-59$ & befriedigend & 4 & $20 \%$ \\
\hline$<40$ & schlecht & 4 & $20 \%$ \\
\hline
\end{tabular}

blutungssituation des Unterschenkels vorhanden ist, wie z. B. bei Patienten mit akutem Trauma ohne Grunderkrankung, um einen möglichst maximalen Längenerhalt der Extremität zu gewährleisten. Da vor der Auswertung der Datenlage des eigenen Patientengutes die Ergebnisse noch nicht vorlagen, wurde die Indikation zur Amputation nach Pirogow wesentlich größer gestellt und auch bei Grunderkrankungen mit mangelhafter Durchblutungssituation durchgeführt, insbesondere da sich in der Literatur keine vergleichbaren Angaben zur Lebensqualität nach Pirogowscher und Unterschenkelamputation fanden. Grundsätzlich ist abzuwägen, ob man den Längenerhalt einer Extremität mit dem größeren Komplikationsrisiko (30\% in der Pirogowgruppe gegenüber $20 \%$ nach Unterschenkelamputation) und der einhergehenden Belastung einer oder mehrerer Folgeoperationen erkaufen soll. Bezüglich der Entscheidung ist nicht nur der Allgemeinzustand und die Compliance des Patienten in Betrachtung zu ziehen, sondern auch kritisch anzumerken, inwieweit eine Gruppengröße von 20 Patienten validierte Daten bezüglich des Komplikationsrisikos zulassen, zumal die multiplen Grunderkrankungen eine eindeutige Aussage verhindern.

10\% der Patienten nach einer Pirogow Amputation geben eine uneingeschränkte Gehstreckenlänge mit Prothese an. 60\% der Patienten geben eine Mobilität ohne Prothese $>10 \mathrm{~m}$ an. Hier ist das Bewertungskriterium des Ankle Scores diskussionswürdig, da bei dieser Antwortoption unklar ist, ob Patienten, die beispielsweise nach erfolgter Amputation im Sprunggelenksbereich $500 \mathrm{~m}$ ohne Prothese laufen können, diese Gehstrecke als uneingeschränkt oder $>10 \mathrm{~m}$ bewerten. Addiert man die Option einer uneingeschränkten Gehstrecke (10\%) mit der Gehstrecke $>10 \mathrm{~m}$ (60\%), so können $70 \%$ der Patienten mehr als $10 \mathrm{~m}$ ohne Prothese zurücklegen, was gerade im häuslichen Bereich einen wesentlichen Vorteil darstellt, da trotz der heutigen besseren prothetischen Versorgung für kurze Wegstrecken - gerade beim nächtlichen Gang zur Toilette - eine Mobilität ohne Prothese erreicht wird. Das Zurücklegen einer gewissen Wegstrecke ohne Prothese ist nach der Unterschenkelamputation dagegen unmöglich [12]. Die Patienten beider Gruppen wurden bei Beantwortung des AS Scores darauf hingewiesen, dass hier explizit die Mobilität ohne Gehstützen bewertet wurde.

Tab. 7 Gunderkrankungen, Indikationen, Komplikationen und Anzahl der Grunderkrankungen Gruppe 2 (Unterschenkel Amputation).

\begin{tabular}{|c|c|c|c|c|}
\hline Patient & Grunderkrankung & Indikation & Komplikation & Anzahl der Revisionseingriffe \\
\hline 1 & keine & Explosionstrauma & keine & 0 \\
\hline 2 & keine & Decollement & keine & 0 \\
\hline 3 & PAVK & Nekrose & keine & 0 \\
\hline 4 & Alkohol und Nikotinabusus, reduzierter AZ & Ulcus cruris & verzögerte Wundheilung & 0 \\
\hline 5 & maligner Knochentumor & Tumor & keine & 0 \\
\hline 6 & Diabetes mellitus & Gangrän & Wundheilungsstörung & 2 (Spalthauttransplantation) \\
\hline 7 & Diabetes mellitus & diabetisches Ulcus & Wunddehiszenz & 5 (VAC) \\
\hline 8 & Nikotin und Alkoholabusus, PAVK & Ulcus cruris & keine & 0 \\
\hline 9 & Nikotin und Alkoholabusus, PAVK & Ulcus cruris & verzögerte Wundheilung & 0 \\
\hline 10 & Alkoholabusus & Verbrennung Grad III & keine & 0 \\
\hline 11 & PAVK & Nekrose & keine & 0 \\
\hline 12 & PAVK & Ulcera & keine & 0 \\
\hline 13 & PAVK & Gangrän & verzögerte Wundheilung & 0 \\
\hline 14 & keine & Explosionstrauma & keine & 0 \\
\hline 15 & Diabetes mellitus & Nekrose & Wunddehiszenz & lokaler Verschiebelappen \\
\hline 16 & keine & Trauma & keine & 0 \\
\hline 17 & Diabetes mellitus & diabetisches Ulcus & Nekrose & 1 (Nachamputation Höhe Oberschenkel) \\
\hline 18 & Diabetes mellitus & Gangrän & verzögerte Wundheilung & 0 \\
\hline 19 & Diabetes mellitus & Ulcera & keine & 1 (Nachamputation Höhe Oberschenkel) \\
\hline 20 & Nikotinabusus, PAVK & Ulcus cruris & keine & 0 \\
\hline
\end{tabular}


Ein weiterer Vorteil der Amputation nach Pirogow ist der Erhalt des plantaren Fußsohlengewebes unter der Ferse als Auftrittsfläche. Das plantare Fett ist mit feinen fibroelastischen Septen in einem 3-dimensionalen Netzwerk durchzogen, welche eine Vielzahl kleiner Dämpfungselemente bilden [8]. Diese einzigartige anatomische Konfiguration macht die Fußsohle zum perfekten Stoßdämpfer während des Ganges, nicht nur für die Mobilität ohne Prothese, sondern auch wenn eine prothetische Versorgung notwendig ist [4]. Der Erhalt dieses Gewebes und somit der Erhalt eines Teils der ursprünglichen Fußsohlensensibilität stellt - gerade da es im Bereich des Unterschenkel Amputationsstumpfes häufig zu rezidivierenden Druckstellen kommt - einen weiteren wesentlichen Vorteil gegenüber der Amputation auf Höhe des Unterschenkels dar [1,15]. Zusätzlich können Patienten mit Verlust der unteren Extremität im Sprunggelenk ohne Prothese schwimmen [11].

Betrachtet man die Resultate der durchgeführten Patientenbefragung mittels AS hinsichtlich des Gesamtergebnisses, so finden sich vergleichbare Ergebnisse der Amputation nach Pirogow und der Unterschenkelamputation. Ein exzellentes bzw. gutes Ergebnis gaben nach Pirogow Amputation 65\% an, nach Unterschenkelamputation $60 \%$.

Auch die Schmerzproblematik ein Jahr nach erfolgter Operation zeigt vergleichbare Resultate. Das funktionelle Ergebnis mit Prothese zeigte in beiden Patientengruppen vergleichbare Ergebnisse, was an den Fortschritten der modernen Prothetik liegt. Die Komplikationsrate der Rückfuß Amputation nach Pirogow kann durch strenge Indikationsstellung möglicherweise weiter reduziert werden. Bei Diabetikern oder Patienten mit mangelhafter Durchblutungssituation der unteren Extremität ist die Amputation auf Höhe des Sprunggelenkes kritisch zu betrachten und sollte nur nach Abwägung der Risiken einer eventuell notwendigen weiteren Amputation auf Höhe des Unterschenkels erfolgen [14]. Kann jedoch eine erfolgreich durchgeführte Amputation auf Höhe des Sprunggelenkes erreicht werden, stellt dies für den Patienten aufgrund der Mobilität ohne Prothese, der einfacheren prothetischen Versorgung und der geringeren $\mathrm{zu}$ leistenden Geharbeit eine wesentlichen Vorteil dar [3].

\section{Fazit für die Praxis}

\section{$\nabla$}

Die Amputation auf Höhe des Sprunggelenkes nach Pirogow stellt bei entsprechender Indikation eine wertvolle Alternative zur Unterschenkelamputation dar. Kann eine erfolgreiche Pirogow Amputation erreicht werden, resultiert für den Patienten eine wesentliche Verbesserung der Lebensqualität.

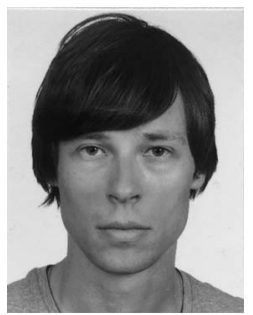

Name: Dr. med Michael Büschges; Geboren: 10.12.1979 in Aachen; Nationalität: deutsch: Familienstand: ledig. 10/2007-12/2007: Assistenzarzt für Augenheilkunde, Universität-Augenklinik Bonn (Prof. Dr. med. F. G. Holz), 01/2008-06/2009: Assistenzarzt für Orthopädie und Unfallchirurgie, Helios Klinik Emil v. Behring, (Prof. Dr. med. J. Scholz),
07/2009-10/2010: Assistenzarzt für Orthopädie und Unfallchirurgie mit Schwerpunkt obere Extremität, Handund Mikrochirurgie, Immanuel Krankenhaus Berlin Wannsee, (Dr. med. Martin Lautenbach).

Seit 12/2010 Assistenzarzt für plastische Chirurgie, Universitätsklinik Lübeck, (Prof. Dr. med. Peter Mailänder). Studienbegleitende Tätigkeiten

Interessenkonflikt: Die Autoren geben an, dass kein Interessenkonflikt besteht.

\section{Literatur}

1 Aitken GT. Surgical amputation. Journal of Bone and Joint Surg 1963; 45: 1735-1741

2 Baumgartner R, Botta P. Amputationschirurgie und Prothesenversorgung. Thieme; Stuttgart: 2007

3 Berlemont M, Weber R, Willot JP. 10 years of Experience with immediate application of prosthetic devices to amputees of the low extremities on the operating table. Prosthet Orthop Int 1969; 3: 8-18

4 Edwards AR. Study Helps Build Functional Bridges for Amputee Patients. Biomechanics 2004; 1: 17-19 [PubMed]

5 Harris RI. Syme's Amputation: the technical details essential for success. J Bone Joint Surg Br 1956; 38: 614

6 Malakhova O. Nikolay Ivanovich Pirogow (1810-1881). Clinical Anatomy 2004; 17: 369-372; [PubMed]

7 Pirogow NI. Questions of Life: Diary of an Old Physician. Science History Publications; Sagamore Beach MA: 1992

8 Steen $M$. Flap surgery for stump improvement of the lower extremity. Fuß \& Sprunggelenk 2007; 5: 113-118; [PubMed]

9 Taniguchi A, Tanaka Y, Kadono $K$ et al. Pirogow ankle disarticulation as an option for ankle disarticulation. Clin Orthop Relat Res 2003; 414: $322-328$

10 Jäger M, Wirth CJ. Praxis in der Orthopädie. Georg Thieme Verlag Stuttgart 1992; 1990-1993

11 Van Damme R, Limet C. Amputation in Diabetic Patients. Clin Pod Med Surg 2007; 24: 569-582; [PubMed]

12 Voloshin I, Bernini PM. Nickolay Ivanovich Pirogow: Innovative Scientist and Clinician. Spine 1998; 23: 2143-2146; [PubMed]

13 Publikation des Wissenschaftlichen Institutes der AOK (WidO), Häufigkeit der Amputationen der unteren Extremität, Erscheinungsjahr 2007

14 Warren G. Amputation of the neuropathic foot - The Pirogow procedure. OP Orth Traum 1997; 9: 460-471; [PubMed]

15 Rammelt S, Olbrich A, Zwipp H. Hindfoot amputations. Oper Orthop Traumatol 2011; 23: 265-279 